\title{
Suppression of the epithelial-mesenchymal transition by SHARP1 is linked to the NOTCH1 signaling pathway in metastasis of endometrial cancer
}

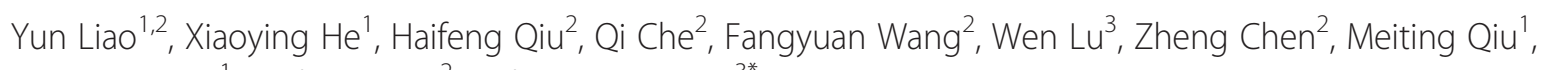
Jingyun Wang ${ }^{1}$, Huihui Wang ${ }^{2}$ and Xiaoping Wan ${ }^{3 *}$

\begin{abstract}
Background: Mechanisms governing the metastasis of endometrial cancer (EC) are poorly defined. Recent data support a role for Enhancer-of-split and hairy-related protein 1 (SHARP1), a basic helix-loop-helix transcription repressor, in regulating invasiveness and angiogenesis of several human cancers. However, the role of SHARP1 in metastasis of EC remains unclear.

Methods: Human EC cell lines (Ishikawa and HEC-1B) were used. SHARP1 was upregulated by lentivirus transduction, while intracellular domain of NOTCH1 (ICN) were upregulated by transient transfection with plasmids. Effects of SHARP1 on cell migration and invasion were evaluated by wound healing assay and transwell invasion assay. Experimental metastasis assay were performed in nude mice. Effects of SHAPR1 on protein levels of target genes were detected by western blotting. Furthermore, the association between SHARP1 and the NOTCH1/EMT pathway was further verified in EC tissue specimens by immunohistochemical analysis.

Results: Overexpression of SHARP1 in EC cells inhibited cell migration, invasion, and metastasis. Exogenous SHARP1 overexpression affected the proteins levels of genes involved in EMT process and NOTCH1 signaling pathway. Upregulation of ICN in SHARP1-overexpressing Ishikawa cells induced cell migration and an EMT phenotype. Additionally, immunohistochemical analysis demonstrated that SHARP1 protein levels were lower in metastatic EC than in primary tumors, and statistical analysis revealed correlations between levels of SHARP1 and markers of EMT and NOTCH1 signaling pathway in human EC tissue specimen.

Conclusions: This work supports a role for SHARP1 in suppressing EMT and metastasis in EC by attenuating NOTCH1 signaling. Therefore, SHARP1 may be a novel marker for lymphatic metastasis in EC patients.
\end{abstract}

Keywords: Endometrial cancer, Metastasis, SHARP1, EMT, NOTCH1

\section{Background}

Endometrial cancer (EC) is the most common gynecological malignancy worldwide. In the United States, it ranks fourth among female malignancies, with an estimated 49,560 new cases and 8190 deaths in 2013 [1]. Despite advances in surgical treatment for early-stage EC (with or without

\footnotetext{
* Correspondence: wanxp@sjtu.edu.cn

${ }^{3}$ Department of Obstetrics and Gynecology, Shanghai First Maternity and Infant Hospital Affiliated to Tong Ji University, No. 536, Changle Road, Shanghai 200080, China

Full list of author information is available at the end of the article
}

adjuvant therapy), treatment of advanced EC is less effective and prognosis is poor [2,3]. The primary reasons for this poor prognosis are metastasis and recurrence, with a median survival of only 7-12 months [4]. It is therefore important to characterize the molecular mechanisms underlying EC metastasis.

Enhancer-of-split and hairy-related protein 1 (SHARP1), which is also called basic helix-loop-helix family, member e41 (BHLHE41) or differentially expressed in chondrocytes 2 (DEC2), is a member of the transcriptional repressor subfamily of basic helix-loop-helix transcription factors 
$[5,6]$ and is expressed in various embryonic and adult tissues $[7,8]$. Emerging evidence suggests that SHARP1 is involved in tumor progression [9-11]. Low-level expression of SHARP1 is associated with the tumor stage in EC [12], and SHARP1 suppresses breast cancer metastasis by degrading hypoxia-inducible factor $1 \alpha$ (HIF-1 $\alpha)$ [11]. In our previous study, we also found that SHARP1 suppresses angiogenesis of endometrial cancer [13]. However, it remains unknown whether SHARP1 contributes to EC metastasis.

Increased cell invasion and migration are defining characteristics of metastatic cancer cells. Recent studies have shown that metastasis can be viewed as a reactivation of at least some aspects of the epithelial-mesenchymal transition (EMT), which normally is an embryonic process [14]. During EMT, epithelial cells undergo extensive alterations in gene expression, resulting in the loss of apical/basolateral polarity, the severing of intercellular adhesive junctions, and the degradation of basement-membrane components. In this way they become individual, non-polarized, motile, and invasive mesenchymal cells [15]. EMT is a dynamic process and is triggered by interactions between extracellular components (such as collagen) and secreted soluble factors (such as the wingless-type MMTV integration site family members (WNTs), transforming growth factor, beta 1, fibroblast growth factors, and epidermal growth factor [14]. Among these signaling pathways, the Notch/Snail/E-cadherin signaling pathway plays a critical role in inducing EMT [16]. High levels of NOTCH1 and its ligand jagged 1 are associated with poor prognosis in breast cancer, bladder cancer, leukemia, prostate cancer [17-19], and EC [20]. In addition, NOTCH1 induces an EMT phenotype and cell migration in pancreatic cancer [21,22] and intrahepatic cholangiocarcinoma [23]. These data prompted us to investigate the mechanisms by which the Notch/EMT signaling pathway is regulated in EC.

Here, we report that SHARP1 inhibits cell migration, invasion, and metastasis in EC cell lines, thereby reverting the EMT cellular phenotype. The effects of SHARP1 on EC involved the NOTCH1 signaling pathway. Re-activation of NOTCH1 signaling in SHARP1 overexpressing EC cells resulted in an EMT phenotype and induced cell migration. Furthermore, the association between SHARP1 and the NOTCH1/EMT pathway was further verified in EC tissue specimens. This work sheds light on the mechanisms and pathways by which EC becomes invasive and metastatic and identifies a potential therapeutic target for treating EC.

\section{Methods}

\section{Ethics statement}

This study was approved by the Human Investigation Ethics Committee of the International Peace Maternity and Child Hospital, which is affiliated with the Shanghai Jiao Tong University School of Medicine. EC specimens were collected after receiving written informed consent from patients. Animal research was carried out in strict accordance with Guideline for the Care and Use of Laboratory Animals of China. The protocol was approved by the Committee on the Ethics of Animal Experiments of the Obstetrical and Gynecological Hospital affiliated Fu Dan University (Permit Number: SYXK (hu) 2008-0064). All efforts were taken to minimize animal suffering.

\section{Patients and samples}

Paraffin-embedded tissue samples were obtained from 15 patients with EC at the International Peace Maternity and Child Health Hospital during 2012 and 2013. The stages (I-IV) and histological grades (G1-G3) of these tumors were established according to criteria of the International Federation of Gynecology and Obstetrics surgical staging system (2009) [24]. None of the patients had undergone hormone therapy, radiotherapy, or chemotherapy before surgery.

\section{Immunohistochemistry (IHC) and assessments}

Tissue sections $(4 \mu \mathrm{m})$ from paraffin-embedded tissue specimens were dewaxed with xylene and then rehydrated using a graded alcohol series. Specimens were then incubated in $0.01 \mathrm{M}$ sodium citrate $(\mathrm{pH} \mathrm{6.0)}$ for $20 \mathrm{~min}$ (for antigen retrieval), $3 \%$ hydrogen peroxide for $10 \mathrm{~min}$ (to block endogenous peroxidase activity), and 10\% normal goat serum for $30 \mathrm{~min}$ (to block nonspecific staining). Sections were then incubated in a humidified chamber at $4^{\circ} \mathrm{C}$ overnight with primary antibodies against the following proteins, from Cell Signaling Technology (Beverly, MA): E-cadherin (1:400), N-cadherin (1:200), vimentin (1:100), and Snail (1:100); from Novus (Littleton, CO): SHARP1 (1:100); and from Abcam (Cambridge, UK): jagged 1 (1:500). Sections incubated with phosphate-buffered solution (PBS) only were used as negative control. Sections were then incubated with biotinylated secondary antibodies for $30 \mathrm{~min}$, followed by streptavidin peroxidase for $15 \mathrm{~min}$. Peroxidase activity was detected by applying 3,3'-diaminobenzidine tetrachloride. Each incubation step was performed at $37^{\circ} \mathrm{C}$ and was followed by three 5 -min washes with PBS. Finally, sections were dehydrated in alcohol and cleared in xylene.

The intensity of IHC staining was scored independently by two pathologists who were blinded to the clinical and pathological data. They used a semiquantitative immunoreactivity score according to Remmele and Stegner [25], which takes into account both the intensity of the color reaction and the percentage of positive cells. The number of positive cells was graded as follows: 0 (<5\%), 1 (5-25\%), 2 (26-50\%), 3 (51-75\%), and 4 (> 75\%). Staining intensity was graded as follows: 0 (negative), 1 (weak), 2 (moderate), and 3 (strong). A final score was calculated by multiplying 
these two scores, generating an immunoreactivity score of $0-12$.

\section{Cell culture and lentivirus transduction}

Human EC cell lines (Ishikawa and HEC-1B) were obtained from the American Type Culture Collection (Manassas, VA). Ishikawa and HEC-1B cells were grown in Dulbecco's modified Eagle medium (DMEM)/F12 (Gibco, Auckland, New Zealand) supplemented with $10 \%$ fetal bovine serum (Gibco, Carlsbad, CA). Cells were cultured in a $5 \% \mathrm{CO}_{2}$ humidified incubator at $37^{\circ} \mathrm{C}$. For stable expression of human SHARP1 in EC cells, SHARP1 coding sequences were cloned into lentiviral vectors with Ubi-MCS-3FLAGSV40-EGFP using Gateway technology (Invitrogen, Carlsbad, CA) by GeneChem Biotech (Shanghai, China).

\section{Transient transfection}

The expression plasmid encoding the intracellular domain of NOTCH1 (ICN), pIRES2-EGFP-ICN, and the empty plasmid, pIRES2-EGFP-NEG, were purchased from R\&S Biotechnology (Shanghai, China). Transient transfection was performed using 70\% confluent Ishikawa cells and Lipofectamine 2000 reagents (Invitrogen).

\section{RNA isolation and quantitative real-time PCR (qPCR)}

Total RNA was extracted from cultured cells using Trizol (Invitrogen). First-strand cDNA was reverse-transcribed from $1 \mu \mathrm{g}$ of total RNA using the Prime Script RT reagent kit (TaKaRa, Dalian, China). The resulting cDNA was analyzed by qPCR using SYBR Premix Ex Taq (TaKaRa). For all qPCR experiments, values on the $y$ axis represent $2^{(-\Delta \mathrm{Ct})}$, where $\Delta \mathrm{Ct}$ is the difference between the geneof-interest $\mathrm{Ct}$ and the $\beta$-actin $\mathrm{Ct}$ [26]. Primer sets are shown in Table 1. Data were obtained in triplicate from three independent experiments.

\section{Western blotting}

Cells were lysed in RIPA lysis buffer (Beyotime, Nanjing, China) with the protease inhibitor phenylmethanesulfonyl fluoride (Beyotime). Protein concentrations were determined using a BCA Protein Assay kit (Beyotime). Equal amounts of protein were loaded into each lane of an SDSPAGE gel. Proteins were then separated with electrophoresis and transferred to a polyvinylidene fluoride membrane (Millipore, Billerica, MA). Each membrane

Table 1 qPCR primer sequences

\begin{tabular}{ll}
\hline Gene & Sequence \\
\hline SHARP1 & Forward 5'-GCATGAAACGAGACGACACC-3' \\
& Reverse 5'-CGCTCCCCATTCTGTAAAGC-3' \\
$\beta$-actin & Forward 5'-CATGTACGTTGCTATCCAGGC-3' \\
& Reverse 5'-CTCCTTAATGTCACGCACGAT-3' \\
\hline
\end{tabular}

was blocked with 5\% skimmed milk for $2 \mathrm{~h}$ and then incubated with antibodies against SHARP1 (1:500), E-cadherin (1:1000), N-cadherin (1:1000), vimentin (1:1000), Snail (1:1000), NOTCH1 (1:2500; Epitomics, Burlingame, CA), jagged 1 (1:10000), HES1 (1:2000; Epitomics), or $\beta$-actin (1:5000; Epitomics) at $4^{\circ} \mathrm{C}$ overnight. Peroxidaselinked secondary antibodies against rabbit (1:5000; Epitomics) were used to detect bound primary antibodies. Probed proteins were detected by enhanced chemiluminescent reagents. The data have been normalized to $\beta$-actin expression by densitometry and statistical data from at least 3 experiments is graphed to provide additional validation of results.

\section{Wound-healing assays}

Cells were grown to confluency as a monolayer and wounded by dragging a $10-\mu \mathrm{L}$ pipette tip through the monolayer. Cells were washed to remove cellular debris and allowed to migrate for $12 \mathrm{~h}$. Representative images were captured at $100 \times$ magnification. All experiments were repeated at least three times.

\section{Trans-well invasion assays}

For trans-well invasion assays, the upper side of an $8-\mu \mathrm{m}$ pore, 6.5-mm polycarbonate trans-well filter (Corning, New York, NY) chamber was uniformly coated with Matrigel basement membrane matrix (BD Biosciences, Bedford, MA) for $2 \mathrm{~h}$ at $37^{\circ} \mathrm{C}$ before cells were added. A total of $5 \times 10^{4}$ cells were seeded into the top chamber of a transwell filter (in triplicate) and incubated for $48 \mathrm{~h}$. Invasive cells, which were on the lower side of the filter, were fixed in $4 \%$ paraformaldehyde, stained in $0.5 \%$ crystal violet (Beyotime), and counted using a microscope. A total of five fields were counted for each trans-well filter. Each field was counted and photographed at $200 \times$ magnification.

\section{In vivo experiments}

Twelve 6-week-old female BALB/c mice were obtained from Shanghai Life Science Institute (Slac Laboratory Animal Co., Ltd., China) and randomly divided into two groups. Each mouse was injected intravenously through the tail vein with $1 \times 10^{6}$ Ishikawa $^{\text {Control }}$ or Ishikawa ${ }^{\text {SHARP1 }}$ cells. Six weeks after the injection, mice were sacrificed and examined.

\section{Statistical analyses}

All statistical analyses were performed using SPSS software, version 17.0 (Chicago, IL). Values represent the mean \pm SD from one representative experiment of three independent experiments, each performed in triplicate. Data was analyzed using the unpaired Student's $t$-test. The spearman's correlation coefficient test was used for correlation detection. A $P$-value of $<0.05$ was considered statistically 
significant. All experiments were performed at least three times.

\section{Results}

SHARP1 inhibits EC-cell migration and invasion in vitro and metastatic potential in vivo

Migration and invasion are important prerequisites for tumor progression and metastasis. To determine the role of SHARP1 in EC progression, we stably transfected EC cell lines with a lentiviral vector expressing human SHARP1. Cells transfected with an empty vector served as the control. These cells lines were named Ishikawa ${ }^{\text {SHARP1 }}$ or Ishikawa ${ }^{\text {Control }}$, and HEC- $1 \mathrm{~B}^{\text {SHARP1 }}$ or HEC- $1 \mathrm{~B}^{\text {Control }}$. Efficient transfection was confirmed before cellular assays were performed (Figure 1A). Wound-healing and transwell invasion assays both demonstrated that the migration and invasion capabilities of Ishikawa and HEC-1B cells were significantly suppressed by SHARP1 overexpression (Figure 1B and C).

To investigate the in vivo effect of SHARP1 on metastasis, we injected Ishikawa cells into severe combined immunodeficiency (SCID) mice. Mice injected with Ishikawa ${ }^{\text {SHARP1 }}$ cells had fewer nodes per lung than mice injected with Ishikawa ${ }^{\text {Control }}$ cells $(4.7 \pm 3.4$ versus $11.8 \pm 3.5, P=0.005)$. Histological studies confirmed that these lesions were caused by the extravasation and subsequent growth of Ishikawa cells in the lungs (Figure 1D). However, no metastasis in other organs was observed (data not shown). Our data indicated that SHARP1 is involved in controlling EC metastasis in vivo.

\section{SHARP1 overexpression reverses the EMT phenotype in EC cells}

Processes involved in the EMT are closely correlated with cancer metastasis. We microscopically examined SHARP1overexpressing EC cells to determine the effects of SHARP1 on cellular morphology. Ishikawa ${ }^{\text {SHARP1 }}$ and HEC-1B ${ }^{\text {SHARP1 }}$ cells were morphologically transformed toward epithelia compared with Ishikawa ${ }^{\text {Control }}$ and HEC-1B ${ }^{\text {Control }}$ cells (Figure 2A). This change was characterized by a loss of spindle-shaped morphology and a gain of cell-cell contacts, suggesting a phenotypic transition from mesenchymal to epithelial. To determine if this morphological transformation represented an EMT (as has been reported [27]), we analyzed levels of several proteins by western blotting. In Ishikawa ${ }^{\text {SHARP1 }}$ and HEC-1B ${ }^{\text {SHARP1 }}$ cells, levels of the epithelial marker E-cadherin were increased, whereas levels of the mesenchymal markers vimentin and $\mathrm{N}$-cadherin were decreased, compared with controls (Figure 2B).

Because E-cadherin is transcriptionally repressed by the transcription factor snail [27], we determined the effect of SHARP1 on snail expression levels. Exogenous
SHARP1 downregulated snail levels in Ishikawa SHARP1 and HEC- $1 \mathrm{~B}^{\text {SHARPI }}$ cells compared with controls (Figure 2B).

\section{SHARP1 overexpression suppresses the NOTCH1 pathway in EC cells}

Activation of the Notch pathway plays a vital role in EMT during cancer progression by transcriptionally activating the gene snail $[28,29]$. We therefore assessed levels of NOTCH1, intracellular domain of NOTCH1 (ICN), its downstream genes HES1and its ligand jagged 1 in Ishikawa and HEC-1B cells. Interestingly, NOTCH1 levels were substantially downregulated by SHARP1 overexpression, as were ICN, HES1 and jagged 1 (Figure 2D). This further supported the hypothesis that SHARP1 inactivates Notch signaling and that the inactivation of Notch signaling might mediate the effect of SHARP1 on the EMT.

\section{Re-activation of NOTCH1 signaling induces migration and an EMT phenotype in SHARP1-overexpressing Ishikawa cells}

To determine whether SHARP1-mediated suppression of the EMT phenotype resulted from SHARP1's ability to inhibit the NOTCH1 pathway, an intracellular domain of NOTCH1 (ICN) was expressed in Ishikawa ${ }^{\text {SHARP1 }}$ cells via transient transfection, which exhibited a constitutively active function of the NOTCH1 receptor. ICN downregulated the epithelial marker E-cadherin, upregulated the mesenchymal markers vimentin and $\mathrm{N}$-cadherin (Figure 3A), and increased the level of Snail and HES1. In addition, ICN promoted the migration of Ishikawa ${ }^{\text {SHARP1 }}$ cells (Figure 3B).

\section{Verification of the SHARP1 effect on the Notch/EMT pathway in EC tissue specimens}

A critical question that arose from our in vitro data was whether SHARP1 levels correlate with metastasis and expression of the EMT markers and Notch1 pathway genes in EC cells, as predicted by our hypothesis. To address this issue, we used a semiquantitative analysis of IHC staining to assess levels of these proteins in 20 specimens from 15 patients with EC. That stands for five specimens from each stage of EC (I-III) and five specimens of metastatic lymph nodes from the very same patients with stage-III EC.

IHC analysis confirmed that SHARP1 levels were significantly lower in metastatic EC tissues $(P=0.0267$; Figure $4 \mathrm{~A}$ and B). Moreover, SHARP1 levels positively correlated with E-cadherin levels $(P=0.0226)$ and inversely correlated with levels of vimentin $(P=0.0391)$, snail $(P=0.0299)$, and jagged $1(P=0.0080)$ (Figure $4 \mathrm{~A}$ and $C)$. And there was a trend of negative correlation between SHARP1 and $\mathrm{N}$-cadherin levels $(P=0.0566)$. 


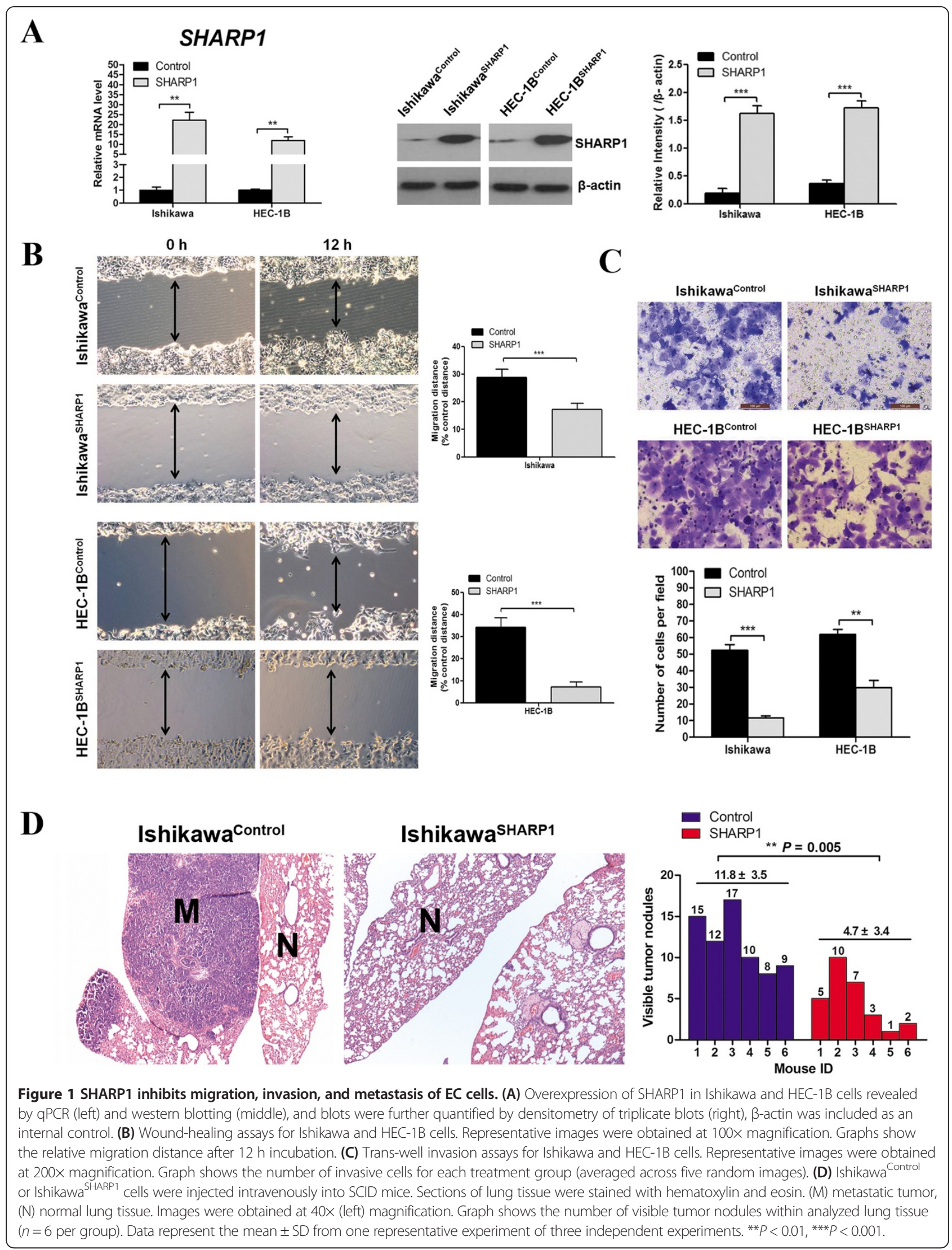



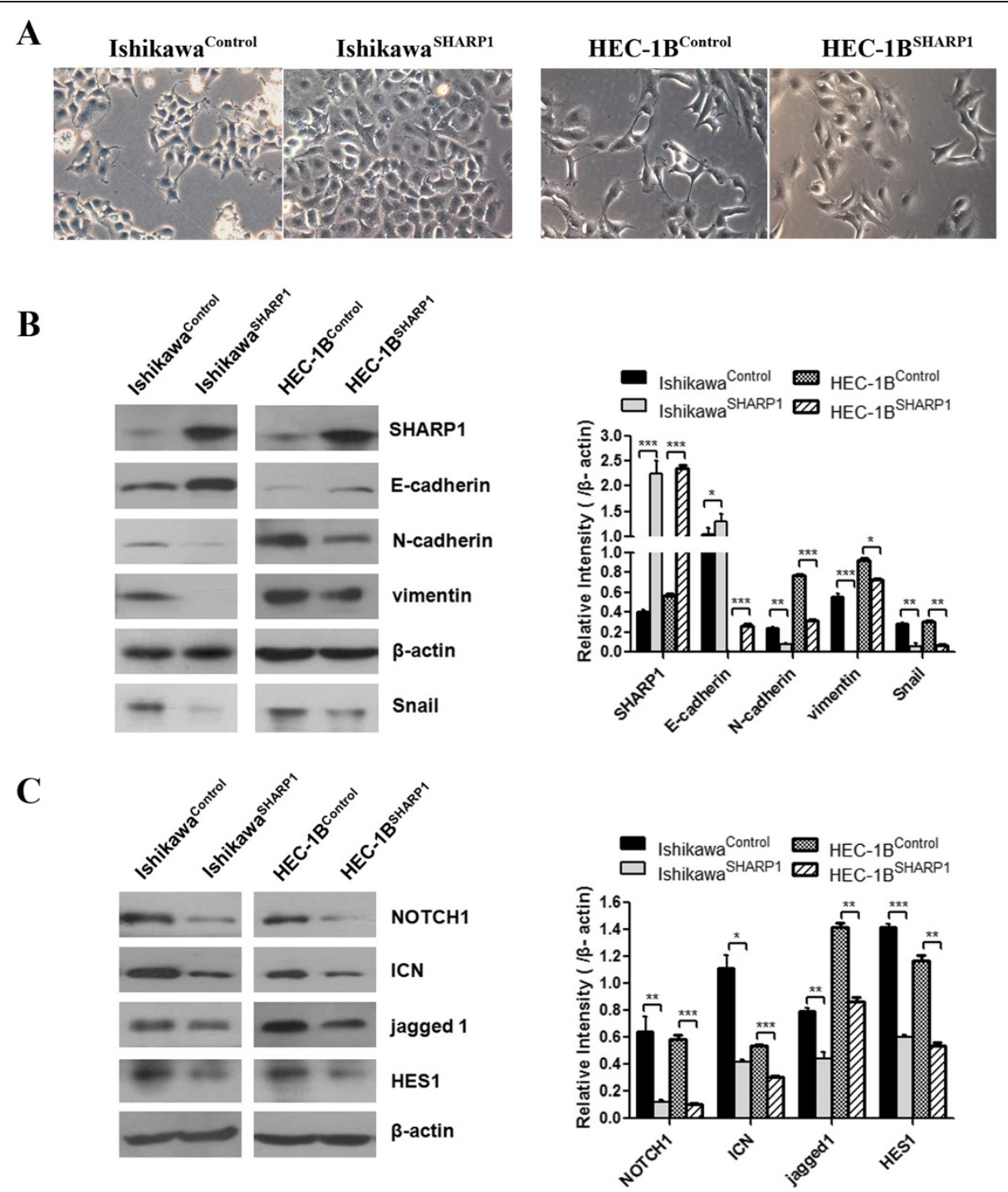

Figure 2 SHARP1 overexpression reverses the EMT phenotype in EC cells. (A) SHARP1 overexpression induced an epithelial morphology in Ishikawa and HEC-1B cells (magnification: 200X). (B) Western blot analysis of SHARP1 and EMT-related markers in Ishikawa and HEC-1B cells (left), and blots were further quantified by densitometry of triplicate blots (right). $\beta$-actin was included as an internal control. (C) Western blot analysis of the effects of SHARP1 overexpression on NOTCH1 signaling components in Ishikawa and HEC-1B cells (left), and blots were further quantified by densitometry of triplicate blots (right). $\beta$-actin was included as an internal control. ${ }^{*} P<0.05,{ }^{* *} P<0.01,{ }^{* * *} P<0.001$.

\section{Discussion}

Tumor invasion and metastasis are complex, multistep processes involving both genetic and epigenetic alterations. Consequently, cancer cells disseminate from the primary tumor and invade distant organs. Because tumor dissemination and metastasis are the leading causes of death in EC, elucidating the molecular mechanisms that underlie invasion and metastasis is important for developing new therapeutic strategies and improving clinical outcomes of patients with EC.

SHARP1 is a basic helix-loop-helix transcription factor that is involved in a number of cellular processes, including proliferation [9], apoptosis [30,31], differentiation [32], and circadian rhythms [33-35]. SHARP1 is generally believed to act as a tumor suppressor, and Montagner, et al. [11] recently showed that SHARP1 regulates the invasive and metastatic phenotype of triple-negative breast cancer cells by promoting degradation of hypoxia-inducible factors. In previous study, we showed SHARP1 suppresses angiogenesis of endometrial cancer by decreasing HIF- $1 \alpha$ level [13]. In the current study, overexpression of SHARP1 suppressed EC-cell migration and invasion in vitro and tumor metastasis in vivo. Our data are consistent with previous findings and suggest for the first time that SHARP1 plays a critical role in tumorigenesis and acquisition of the metastatic phenotype in EC. However, the underlying 

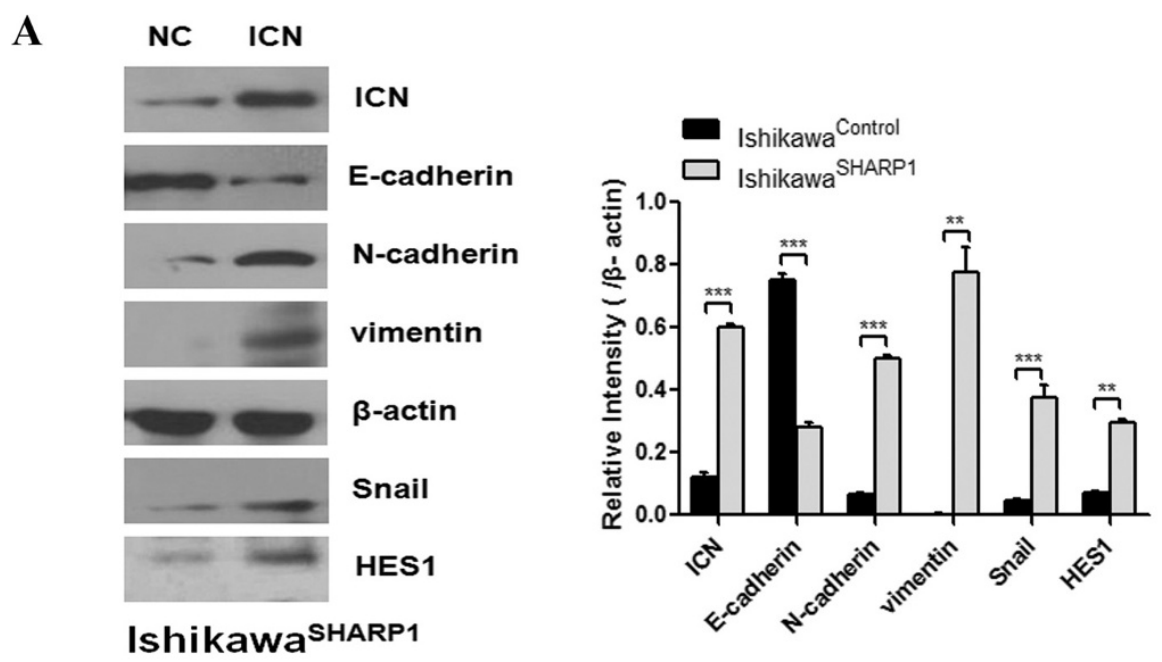

B
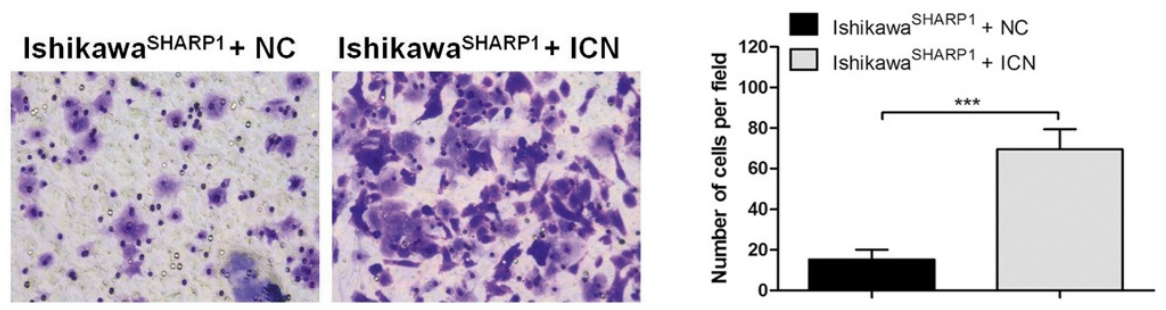

Figure 3 Signaling through the NOTCH1 pathway induces migration and an EMT phenotype in SHARP1-overexpressing Ishikawa cells. (A) Western blot analysis of Ishikawa ${ }^{\text {SHARP1 }}$ cells expressing the intracellular domain of NOTCH1 (ICN) or a negative control (NC). Protein levels of ICN, HES1 and EMT-related markers were analyzed by western blotting in Ishikawa ${ }^{\text {SHARP1 }}$ cells (Left), and further quantified by densitometry of triplicate blots (right). $\beta$-actin was included as an internal control. (B) Trans-well migration assays involving Ishikawa ${ }^{\text {SHARP1 }}$ cells expressing ICN or NC. Representative images were obtained at 200x magnification. Graph shows the average number of migrated cells ( $n=5$ images) for the two treatment groups. Data represent the mean \pm SD from one representative experiment of three independent experiments. ${ }^{* *} P<0.01,{ }^{* *} P<0.001$.

mechanisms remain unknown and must be addressed by further investigation.

The EMT is a key event during cancer progression, leading to a more invasive, metastatic phenotype in human cancers, including EC [14,29]. At the molecular level, a variety of factors have been implicated in EMT. The loss of E-cadherin appears to be a crucial step, as this reduces cell-cell adhesion and destabilizes the epithelial architecture. Moreover, the protein snail, which is activated during the acquisition of EMT, plays a central role in repressing E-cadherin expression. Recent studies indicate that EMT status is associated with aggressive tumor characteristics and prognosis in EC [36,37]. However, the role of SHARP1 in this process remains unclear. Here we observed a morphological transformation in Ishikawa ${ }^{\text {SHARP1 }}$ and HEC- $1 \mathrm{~B}^{\text {SHARP1 }}$ cells with a concomitant increase in $\mathrm{E}$-cadherin and reduction in $\mathrm{N}$-cadherin and vimentin, suggesting a mesenchymal-to-epithelial transition in EC. Moreover, the reduced level of snail in these cells supports the idea that SHARP1 inhibits EMT in EC.

The Notch pathway is highly conserved and regulates cell fate specification, stem cell maintenance, and the initiation of differentiation in embryonic and postnatal tissues. Notch signaling also promotes EMT during cardiac development and tumor progression by inducing Snail, which subsequently downregulates cadherins [28]. Our data showed that the expression of NOTCH1 signaling pathway genes, Notch1, ICN, jagged 1 and HES1, was attenuated by SHARP1 overexpression in EC cells, indicating that SHARP1 inactivated the NOTCH1 pathway and suggesting that SHARP1-mediated suppression of NOTCH1 signaling contributes to suppression of EMT in EC. Future studies must determine whether SHARP1 regulates other signaling pathways capable of inducing EMT, such as the $\mathrm{NF} \kappa \mathrm{B}$ and $\mathrm{WNT} / \beta$-catenin pathways.

Tumor-cell metastasis and invasion are responsible for most cancer-related mortalities. Invasive EC cells primarily metastasize to the lymphatic system, and we showed that SHARP1 overexpression decreased lymph-node metastases compared with primary tumors. Moreover, we detected a positive correlation between SHARP1 and E-cadherin levels and negative correlations between SHARP1 level and levels of vimentin, snail, and jagged 1. These results support our findings in EC cell lines. Although surgery is 


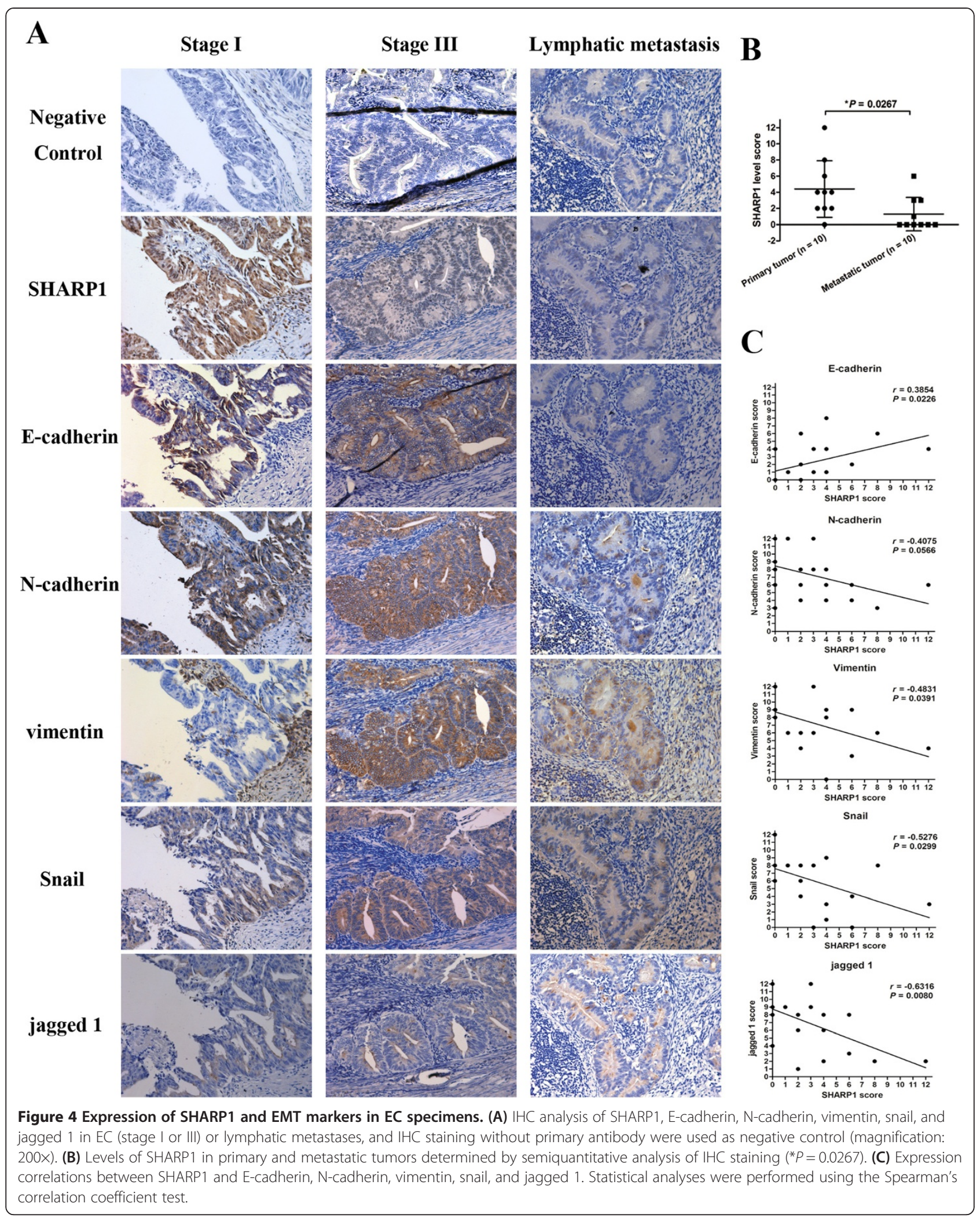


the standard treatment for patients with EC, patients with lymph-node or distant-organ metastases also require chemoradiotherapy. Thus, identifying biomarkers that define the metastatic potential of EC cells may help optimize treatment strategies. In this study, we found that evaluating SHARP1 levels in EC tissues (using IHC) effectively identified patients with EC who were at risk for lymph-node metastasis. Thus, SHARP1 may be a potential marker for EC metastasis and a target for therapeutic intervention.

\section{Conclusions}

In summary, our results with EC cells show that SHARP1 suppressed migration, invasion, the EMT phenotype, and metastasis and that these effects involved downregulation of NOTCH1 signaling. Moreover, using EC tissue specimens we detected negative correlations between SHARP1 level and both lymphatic metastasis and markers of EMT. Our data suggest for the first time that impacts of SHARP1 on the NOTCH1/EMT system play a critical role in malignant progression and acquisition of metastatic phenotypes in EC. Thus, targeting SHARP1 could represent a new treatment option for preventing EC metastasis.

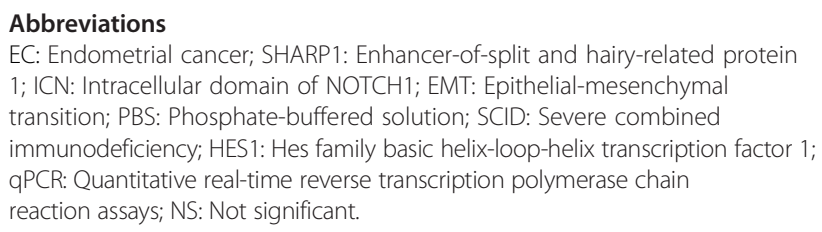

\section{Competing interests}

The authors have declared that no competing interests exist.

\section{Authors' contributions}

$Y L, X H$, and $H Q$ carried out the design of the experiments, performed most of experiments, and drafted the manuscript. ZC, WL and HW participated in the molecular biology experiments and statistical analysis. QC and FW participated in tumor pathological characterization. JW and MQ made the figures. XW was involved in financial support, the design of the experiments, data analysis, and final approval of the manuscript. All authors read and approved the final manuscript.

\section{Acknowledgments \\ We thank Gufeng Xu, Qin Huang and Fuju Tian (Center Laboratory of International Peace Maternity \& Child Health Hospital affiliated to Shanghai Jiao Tong University School of Medicine, Shanghai, China) for excellent technical support. This study was supported by the National Natural Science Foundation of China $(81272885,81172476)$, the Foundation Project of Shanghai Municipal Science and Technology Commission (No. 13JC1404500) and the Ph. D. Programs Foundation of the Ministry of Education of China (No. 2012007211090).}

\section{Author details}

${ }^{1}$ Department of Obstetrics and Gynecology, International Peace Maternity \& Child Health Hospital Affiliated to Shanghai Jiao Tong University School of Medicine, Shanghai, China. ${ }^{2}$ Department of Obstetrics and Gynecology, Shanghai First People's Hospital Affiliated to Shanghai Jiao Tong University School of Medicine, Shanghai, China. ${ }^{3}$ Department of Obstetrics and Gynecology, Shanghai First Maternity and Infant Hospital Affiliated to Tong Ji University, No. 536, Changle Road, Shanghai 200080, China.

Received: 25 February 2014 Accepted: 30 June 2014 Published: 5 July 2014

\section{References}

1. Siegel R, Naishadham D, Jemal A: Cancer statistics, 2013. CA Cancer J Clin 2013, 63(1):11-30.

2. Di Cristofano A, Ellenson LH: Endometrial carcinoma. Annu Rev Pathol 2007, 2:57-85.

3. Hill EK, Dizon DS: Medical therapy of endometrial cancer: current status and promising novel treatments. Drugs 2012, 72(5):705-713.

4. Oza AM, Elit L, Tsao MS, Kamel-Reid S, Biagi J, Provencher DM, Gotlieb WH Hoskins PJ, Ghatage P, Tonkin KS, Mackay HJ, Mazurka J, Sederias J, Ivy P, Dancey JE, Eisenhauer EA: Phase II study of temsirolimus in women with recurrent or metastatic endometrial cancer: a trial of the NCIC Clinical Trials Group. J Clin Oncol 2011, 29(24):3278-3285.

5. Fujimoto K, Shen M, Noshiro M, Matsubara K, Shingu S, Honda K, Yoshida E, Suardita K, Matsuda Y, Kato Y: Molecular cloning and characterization of DEC2, a new member of basic helix-loop-helix proteins. Biochem Biophys Res Commun 2001, 280(1):164-171.

6. Fujimoto K, Hamaguchi H, Hashiba T, Nakamura T, Kawamoto T, Sato F, Noshiro M, Bhawal UK, Suardita K, Kato Y: Transcriptional repression by the basic helix-loop-helix protein Dec2: multiple mechanisms through E-box elements. Int J Mol Med 2007, 19(6):925-932.

7. Azmi S, Taneja R: Embryonic expression of mSharp-1/mDEC2, which encodes a basic helix-loop-helix transcription factor. Mech Dev 2002 114(1-2):181-185.

8. Azmi S, Sun H, Ozog A, Taneja R: mSharp-1/DEC2, a basic helix-loop-helix protein functions as a transcriptional repressor of $E$ box activity and Stra13 expression. J Biol Chem 2003, 278(22):20098-20109.

9. Bhawal UK, Sato F, Arakawa Y, Fujimoto K, Kawamoto T, Tanimoto K, Ito Y, Sasahira T, Sakurai T, Kobayashi M, Kashima I, Kijima H, Kuniyasu H, Abiko Y, Kato Y, Sato S: Basic helix-loop-helix transcription factor DEC1 negatively regulates cyclin D1. J Pathol 2011, 224(3):420-429.

10. Li Y, Shen Q, Kim HT, Bissonnette RP, Lamph WW, Yan B, Brown PH: The rexinoid bexarotene represses cyclin D1 transcription by inducing the DEC2 transcriptional repressor. Breast Cancer Res Treat 2011, 128(3):667-677.

11. Montagner M, Enzo E, Forcato M, Zanconato F, Parenti A, Rampazzo E, Basso G, Leo G, Rosato A, Bicciato S, Cordenonsi M, Piccolo S: SHARP1 suppresses breast cancer metastasis by promoting degradation of hypoxia-inducible factors. Nature 2012, 487(7407):380-384.

12. Yunokawa M, Tanimoto K, Nakamura H, Nagai N, Kudo Y, Kawamoto T Kato Y, Hiyama E, Hiyama K, Nishiyama M: Differential regulation of DEC2 among hypoxia-inducible genes in endometrial carcinomas. Oncol Rep 2007, 17(4):871-878.

13. Liao Y, Lu W, Che Q, Yang T, Qiu H, Zhang H, He X, Wang J, Qiu M, Zou Y, Gu W, Wan X: SHARP1 Suppresses Angiogenesis of Endometrial Cancer by Decreasing Hypoxia-Inducible Factor-1alpha Level. PLoS One 2014, 9(6):e99907.

14. Yang J, Weinberg RA: Epithelial-mesenchymal transition: at the crossroads of development and tumor metastasis. Dev Cell 2008, 14(6):818-829.

15. Lee JM, Dedhar S, Kalluri R, Thompson EW: The epithelial-mesenchymal transition: new insights in signaling, development, and disease. J Cell Biol 2006, 172(7):973-981.

16. Sahlgren C, Gustafsson MV, Jin S, Poellinger L, Lendahl U: Notch signaling mediates hypoxia-induced tumor cell migration and invasion. Proc Natl Acad Sci U S A 2008, 105(17):6392-6397.

17. Zhu YM, Zhao WL, Fu JF, Shi JY, Pan Q, Hu J, Gao XD, Chen B, Li JM, Xiong SM, Gu LJ, Tang JY, Liang H, Jiang H, Xue YQ, Shen ZX, Chen Z, Chen SJ: NOTCH1 mutations in T-cell acute lymphoblastic leukemia: prognostic significance and implication in multifactorial leukemogenesis. Clin Cancer Res 2006, 12(10):3043-3049.

18. Reedijk M, Pinnaduwage D, Dickson BC, Mulligan AM, Zhang H, Bull SB, O'Malley FP, Egan SE, Andrulis IL: JAG1 expression is associated with a basal phenotype and recurrence in lymph node-negative breast cancer. Breast Cancer Res Treat 2008, 111(3):439-448.

19. Shi TP, Xu H, Wei JF, Ai X, Ma X, Wang BJ, Ju ZH, Zhang GX, Wang C, Wu ZQ, Zhang $X$ : Association of low expression of notch-1 and jagged-1 in human papillary bladder cancer and shorter survival. J Urol 2008, 180(1):361-366.

20. Mitsuhashi Y, Horiuchi A, Miyamoto T, Kashima H, Suzuki A, Shiozawa T: Prognostic significance of Notch signalling molecules and their involvement in the invasiveness of endometrial carcinoma cells. Histopathology 2012, 60(5):826-837.

21. Wang Z, Li Y, Kong D, Banerjee S, Ahmad A, Azmi AS, Ali S, Abbruzzese JL, Gallick GE, Sarkar FH: Acquisition of epithelial-mesenchymal transition 
phenotype of gemcitabine-resistant pancreatic cancer cells is linked with activation of the notch signaling pathway. Cancer Res 2009, 69(6):2400-2407.

22. Bao B, Wang Z, Ali S, Kong D, Li Y, Ahmad A, Banerjee S, Azmi AS, Miele L, Sarkar FH: Notch-1 induces epithelial-mesenchymal transition consistent with cancer stem cell phenotype in pancreatic cancer cells. Cancer Lett 2011, 307(1):26-36.

23. Zhou $Q$, Wang $Y$, Peng $B$, Liang $L$, Li J: The roles of Notch1 expression in the migration of intrahepatic cholangiocarcinoma. BMC Cancer 2013, 13:244.

24. Creasman W: Revised FIGO staging for carcinoma of the endometrium. Int J Gynaecol Obstet 2009, 105(2):109.

25. Remmele W, Stegner HE: Recommendation for uniform definition of an immunoreactive score (IRS) for immunohistochemical estrogen receptor detection (ER-ICA) in breast cancer tissue. Pathologe 1987, 8(3):138-140.

26. Yang T, Qiu H, Bao W, Li B, Lu C, Du G, Luo X, Wang L, Wan X: Epigenetic inactivation of EFEMP1 is associated with tumor suppressive function in endometrial carcinoma. PLoS One 2013, 8(6):e67458.

27. Bao W, Qiu H, Yang T, Luo X, Zhang H, Wan X: Upregulation of TrkB Promotes Epithelial-Mesenchymal Transition and Anoikis Resistance in Endometrial Carcinoma. PLoS One 2013, 8(7):e70616.

28. Timmerman LA, Grego-Bessa J, Raya A, Bertrán E, Pérez-Pomares JM, Díez J, Aranda S, Palomo S, McCormick F, Izpisúa-Belmonte JC, de la Pompa JL: Notch promotes epithelial-mesenchymal transition during cardiac development and oncogenic transformation. Genes Dev 2004, 18(1):99-115.

29. Wang Z, Li Y, Kong D, Sarkar FH: The role of Notch signaling pathway in epithelial-mesenchymal transition (EMT) during development and tumor aggressiveness. Curr Drug Targets 2010, 11(6):745-751.

30. Liu Y, Sato F, Kawamoto T, Fujimoto K, Morohashi S, Akasaka H, Kondo J, Wu Y, Noshiro M, Kato Y, Kijima H: Anti-apoptotic effect of the basic helix-loop-helix (bHLH) transcription factor DEC2 in human breast cancer cells. Genes Cells 2010, 15(4):315-325.

31. Wu Y, Sato F, Bhawal UK, Kawamoto T, Fujimoto K, Noshiro M, Seino H, Morohashi S, Kato Y, Kijima H: BHLH transcription factor DEC2 regulates pro-apoptotic factor Bim in human oral cancer HSC-3 cells. Biomed Res 2012, 33(2):75-82.

32. Gulbagci NT, Li L, Ling B, Gopinadhan S, Walsh M, Rossner M, Nave KA, Taneja R: SHARP1/DEC2 inhibits adipogenic differentiation by regulating the activity of C/EBP. EMBO Rep 2009, 10(1):79-86.

33. Rossner MJ, Oster H, Wichert SP, Reinecke L, Wehr MC, Reinecke J, Eichele G Taneja R, Nave KA: Disturbed clockwork resetting in Sharp-1 and Sharp-2 single and double mutant mice. PLoS One 2008, 3(7):e2762.

34. Sato F, Bhawal UK, Kawamoto T, Fujimoto K, Imaizumi T, Imanaka T, Kondo J, Koyanagi S, Noshiro M, Yoshida H, Kusumi T, Kato Y, Kijima H: Basic-helix-loophelix (bHLH) transcription factor DEC2 negatively regulates vascular endothelial growth factor expression. Genes Cells 2008, 13(2):131-144.

35. Hamaguchi H, Fujimoto K, Kawamoto T, Noshiro M, Maemura K, Takeda N, Nagai R, Furukawa M, Honma S, Honma K, Kurihara H, Kato Y: Expression of the gene for Dec2, a basic helix-loop-helix transcription factor, is regulated by a molecular clock system. Biochem J 2004, 382(Pt 1):43-50.

36. Zhang J, Wei J, Lu J, Tong Z, Liao B, Yu B, Zheng F, Huang X, Chen Z, Fang Y, Li B, Chen W, Xie D, Luo J: Overexpression of Rab25 contributes to metastasis of bladder cancer through induction of epithelial-mesenchymal transition and activation of Akt/GSK-3beta/Snail signaling. Carcinogenesis 2013, 34(10):2401-2408.

37. Tanaka Y, Terai Y, Kawaquchi H, Fujiwara S, Yoo S, Tsunetoh S, Takai M, Kanemura M, Tanabe A, Ohmichi M: Prognostic impact of EMT (epithelial-mesenchymal-transition)-related protein expression in endometrial cancer. Cancer Biol Ther 2013, 14(1):13-19.

doi:10.1186/1471-2407-14-487

Cite this article as: Liao et al: Suppression of the epithelial-mesenchymal transition by SHARP1 is linked to the NOTCH1 signaling pathway in metastasis of endometrial cancer. BMC Cancer 2014 14:487.

\section{Submit your next manuscript to BioMed Central and take full advantage of:}

- Convenient online submission

- Thorough peer review

- No space constraints or color figure charges

- Immediate publication on acceptance

- Inclusion in PubMed, CAS, Scopus and Google Scholar

- Research which is freely available for redistribution

Submit your manuscript at www.biomedcentral.com/submit
C BioMed Central 